\title{
Intoxicações agudas no serviço de urgência
}

\author{
Acute intoxications in the emergency department \\ Marta Oliveira Ferreira, Diana Fernandes, Liliana Oliveira, Maria Vilela,
Tatiana Salazar, David Silva, Nuno Cardoso, Augusto Duarte
Centro Hospitalar do Médio Ave, Famalicão (Portugal)
}

\begin{abstract}
Acute poisoning are a frequent cause of recurring to emergency departments. This study aims to characterize poisoning in one emergency department. We made a retrospective study of the admissions to the emergency department of Famalicão and Santo Tirso's units of Centro Hospitalar do Médio Ave in Portugal for 6 months.

The 125 selected patients, 63 men and 62 women, had a mean age of 46.7 years. The intoxication was voluntary in 99 patients $(79.2 \%)$ and accidental in 26 patients (20.8\%). Ethanol, benzodiazepines and antipsychotics were the three most frequent toxic groups. There was contact with more than one type of toxic in 32 cases (25.6\%). On average, patients remained 9h10minutes in emergency department. There were no complications in 108 patients. The most common destinations were the family doctor and the assessment by psychiatry.

This study allowed us to know the reality of this emergency service, which will facilitate the approach of acute poisoning. For more information about the poisonings in Portugal, should be performed prospective multicenter studies in order to understand consumption patterns, to know the more often involved substances and the associated complications, and prepare preventive action plans in the community.
\end{abstract}

Palabras clave: Envenenamiento. Emergencias. Sustancias tóxicas.

\section{Introdução}

Na prática clínica, o conceito de intoxicação corresponde ao contacto com substâncias que, na dependência das suas propriedades físicas ou químicas e da magnitude da exposição, são passíveis de perturbar o normal funcionamento do organismo. ${ }^{1}$

As intoxicações agudas constituem um sério problema de saúde, sendo um motivo frequente de admissão nos serviços de urgência (SU). ${ }^{2}$ Independentemente da sua causa (que engloba a intenção suicida, abuso de substâncias ou acidentes), a mortalidade a longo prazo dos doentes vítimas de intoxicação é elevada, o que sublinha a necessidade do conhecimento deste tipo de situações e do seu impacto social. ${ }^{2}$

A sua abordagem pode começar no local do contacto com o tóxico, durante o transporte para uma unidade de saúde ou no hospital. ${ }^{3}$

Os médicos generalistas são frequentemente os primeiros a abordar as vítimas de intoxicação, pelo que devem estar aptos a prestar o tratamento inicial adequado, sendo importante ter experiência na abordagem destas situações e ter rápido acesso à informação toxicológica. ${ }^{3}$

\section{Resumo}

As intoxicações agudas são motivo frequente de recorrência aos serviços de urgência. Este estudo pretende caracterizar as intoxicações num serviço de urgência.Fez-se o estudo retrospetivo das admissões no serviço de urgência das Unidades de Famalicão e Santo Tirso do Centro Hospitalar do Médio Ave, em Portugal, durante 6 meses.

Os 125 doentes selecionados, 63 homens e 62 mulheres, tinham idade média de 46,7 anos. A intoxicação foi voluntária em 99 doentes (79.2\%) e acidental em 26 doentes (20,8\%). 0 etanol, as benzodiazepinas e os antipsicóticos foram os três grupos de tóxicos mais frequentes. Houve contacto com mais do que um tipo de tóxico em 32 casos (25,6\%). Em média, os doentes permaneceram 09h10 na urgência. Não houve complicações em 108 indivíduos. Os destinos de alta mais frequentes foram o médico de família e a avaliação por psiquiatria.

Este estudo permitiu conhecer a realidade deste serviço de urgência 0 que facilitará a abordagem das intoxicações agudas. Para obter mais informação acerca das intoxicações em Portugal deverão ser realizados estudos prospetivos multicêntricos, por forma a conhecer padrões de consumo, substâncias mais vezes implicadas e tipo de complicações associadas, e elaborar planos preventivos de ação na comunidade.

Keywords: Poisoning. Emergencies. Toxic substances.

Existem diferenças nos padrões de intoxicação de centros de diferentes áreas geográficas, tendo havido também variações ao longo dos tempos. ${ }^{2,4,5}$ Surgem novas drogas, alteram-se os padrões de intoxicação por substâncias familiares, existem endemias locais e epidemias. ${ }^{2} \mathrm{~A}$ época do ano também parece influenciar a incidência das intoxicações, cuja média é mais alta nos meses mais quentes. ${ }^{6}$

De acordo com o relatório da American Association of Poison Control Centers, o número de intoxicações no ano 2014 foi de 6,7 por 1000 habitantes. ${ }^{7}$ Em Portugal, os dados publicados pelo Centro de Informação Antivenenos (CIAV) referentes ao ano 2014 apontam para 32232 chamadas entre janeiro e dezembro desse ano, 7689 das quais realizadas por profissionais de saúde. ${ }^{8}$ De acordo com a mesma fonte, os medicamentos foram os principais agentes envolvidos nas intoxicações em adultos (21656), seguidos dos domésticos/ industriais (5290), pesticidas (2141), substâncias de abuso (982), cosméticos (775), escolares/brinquedos (282), plantas (120) e cogumelos (49). ${ }^{8}$

Para manter actualizado o conhecimento destas novas tendências e mudanças, são necessários estudos da realidade de cada serviço de urgência. ${ }^{2}$ 
Este estudo tem por objetivo caracterizar as intoxicações admitidas num SU, dando ênfase ao tipo de substâncias implicadas, tratamentos realizados, complicações e orientação.

\section{Material e métodos}

Procedeu-se ao estudo retrospetivo dos doentes admitidos no SU das Unidades de Famalicão e Santo Tirso do Centro Hospitalar do Médio Ave, em Portugal, no período de 6 meses (entre 1 de Julho e 31 de dezembro de 2015). Trata-se de um hospital distrital com uma população de referência de cerca de 240000 pessoas, e uma lotação de 247 camas, que não dispões de unidade de cuidados intensivos. Excluíram-se as admissões na urgência pediátrica.

Foram selecionados os doentes cujos diagnósticos de alta, codificados de acordo com ICD-9, incluíram os seguintes códigos:

- 960 a 979 (Envenenamento por medicamentos e substâncias biológicas).

- 980 a 989 (Efeitos tóxicos de substâncias que não sejam medicamentos).

- E950, E951 e E952 (Suicídio e envenenamento auto-infligido por substâncias líquidas ou sólidas; gases de uso doméstico; outros gases e vapores).

- 292.21 (Intoxicação patológica por drogas).

Para cada doente selecionado, foi consultado o processo clínico eletrónico para o registo dos seguintes dados:

- género;

- idade;

- prioridade de triagem de acordo com o sistema de Manchester;

- tipo de assistência pré-hospitalar, se existente;

- tipo e quantidade do tóxicos implicado;

- tipo de Intoxicação (voluntária versus acidental);

- tratamento realizado;

- complicações verificadas;

- hora de admissão e alta do SU;

- destino de alta.

A análise estatística dos resultados foi feita com recurso ao programa Microsoft Office Excel 2013 ${ }^{\circledR}$.

\section{Resultados}

Cumpriram os critérios de seleção 125 doentes, correspondentes a $0,29 \%$ do total de episódios de urgência de adultos em igual período.

Dos doentes estudados, 63 eram do género masculino e 62 do género feminino, sendo a idade média de 46,7 anos (mínimo de 16 anos; máximo de 93 anos).

Seis doentes foram assistidos pela Viatura Médica de Emergência e Reanimação (VMER), três doentes foram assistidos por ambulância de Suporte Imediato de Vida (SIV) e os restantes 116 doentes (92,8\% da amostra) foram trazidos ao SU por corporações de bombeiros ou por meios próprios.
Em 99 doentes (79.2\%) a intoxicação foi voluntária, enquanto nos restantes 26 doentes (20,8\%) a intoxicação foi acidental. Analisando separadamente por género, constatou-se que para o género feminino os três grupos de tóxicos mais frequentes foram as benzodiazepinas (n 37; 59,7\%), os inibidores seletivos da recaptação da serotonina (SSRI) (n 8; 12,9\%) e os antipsicóticos (n 8; 12,9\%). No género masculino os três grupos de tóxicos mais frequentes foram o etanol (n $49 ; 77,8 \%$ ), seguido das benzodiazepinas (n $6 ; 9,5 \%$ ) e dos antipsicóticos (n 5; 7,9\%).

Em média, observou-se a ingestão de 1,48 tipos de tóxicos (mínimo 1 tipo; máximo de 5 tipos). Perto de um quarto dos doentes (n 32; 25,6\%) tiveram contacto com mais do que um tipo de tóxico.

Em média, os doentes permaneceram 09h10 no SU (mínimo de permanência 21 minutos; máximo 52h04). 0 tempo de demora no SU por intervalos é apresentado na Figura 3.

Os destinos de alta desta amostra foram: médico de família (66/52,8\%), serviço de psiquiatria $(36 / 28,8 \%)$, saída contra parecer $(9 / 7,2 \%)$, área de observação do SU $(7 / 5,6 \%)$, unidade de cuidados intensivos $(3 / 2,4 \%)$, serviço de medicina $(3 / 2,4 \%)$ e abandono (1/0,8\%).

No que concerne ao tratamento, em 70 casos (56\%), não existia antídoto para os tóxicos implicados. Nos restantes casos, 0 antídoto foi administrado em 36 doentes, mas não 0 foi em 19 casos. A lavagem gástrica foi realizada em 91 doentes (72,8\%) e o carvão ativado administrado em 96 casos (76,8\%).

Não houve menção a complicações em 116 dos doentes, mas quatro doentes apresentaram instabilidade hemodinâmica, três tiveram complicações neurológicas e dois necessitaram de entubação orotraqueal.

\section{Discussão e conclusão}

Conhecer a realidade do SU onde se trabalha é fundamental para otimizar a abordagem das vítimas de intoxicação.

0 presente estudo permitiu perceber padrões de comportamento, identificar as substâncias mais vezes implicadas nas intoxicações e as complicações associadas.

Embora a população estudada tenha visado apenas as admissões no SU de adultos, foi incluído um doente de 16 anos que recorreu ao SU da unidade de Santo Tirso, na qual não existe urgência pediátrica.

As intoxicações foram maioritariamente voluntárias, à semelhança dos dados reportados em estudos semelhantes, como o realizado por Kaya $E$ e colaboradores num serviço de urgência de um hospital universitário na Turquia. ${ }^{4}$

0 etanol e os medicamentos destacaram-se como os tóxicos mais frequentemente implicados, o que pode traduzir 0 fácil acesso a este tipo de substâncias. Estes dados são concordantes com o relatório do CIAV do ano 2014, no qual os medicamentos encabeçaram os grupos de tóxicos mais frequentemente assinalados. ${ }^{8}$ 
Fig 1. Apresenta as prioridades de triagem desta amostra de doentes, de acordo com o sistema de Manchester.

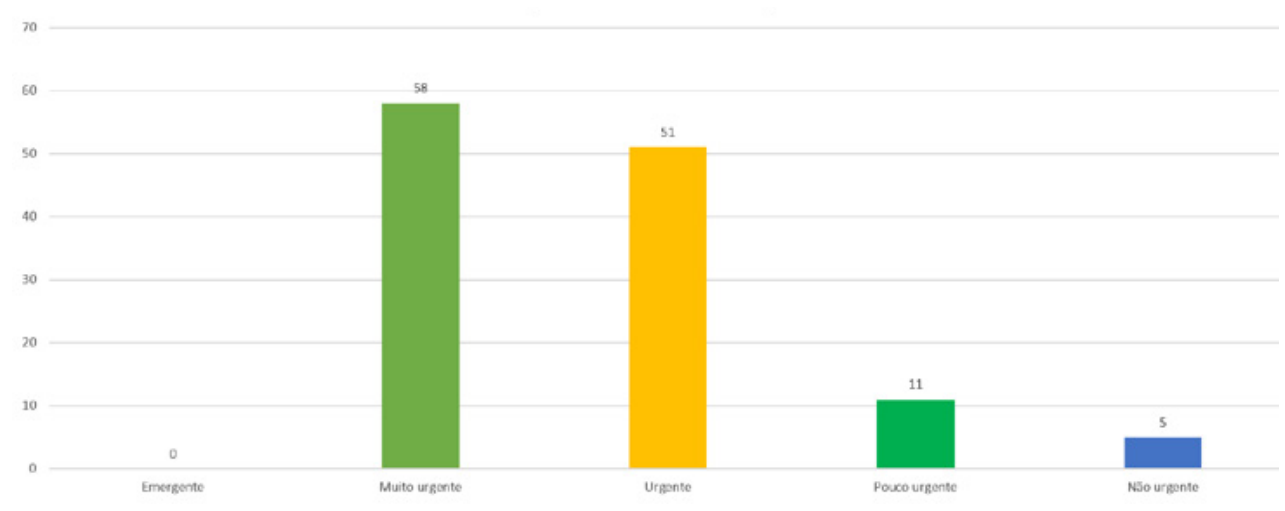

Tabela 1. Estão listados os grupos de tóxicos implicados.

\begin{tabular}{|lcc|}
\hline \multicolumn{1}{|c|}{ Grupo farmacológico } & Tabela 1 - Frequência dos tóxicos por classes & $\%$ \\
\hline Etanol & $n$ & 44,8 \\
\hline Benzodiazepinas & 56 & 34.4 \\
\hline Antipsicóticos & 43 & 10,4 \\
\hline Inibidores da Recaptação da Serotonina & 13 & 8,8 \\
\hline Anti-hipertensores & 11 & 4,0 \\
\hline Paracetamol & 5 & 4,0 \\
\hline Digoxina & 5 & 4,0 \\
\hline Anti-inflamatórios Não Esteróides & 4,0 \\
\hline Antidepressivos tricíclicos & 5 & 3,2 \\
\hline Anti-epiléticos & 5 & \\
\hline Mercúrio & 2,4 \\
\hline Inibidores da Mono-aminoxidase & 1,6 \\
\hline Organofosforados & 4 & 1,6 \\
\hline Estatinas & 3 & 1,6 \\
\hline Valeriana & 2 & 1,6 \\
\hline Antibióticos & 2 & 1,6 \\
\hline Opióides & 2 & 1,6 \\
\hline Outros & 2 & 1,6 \\
\hline
\end{tabular}

Fig 2. Apresentam-se o número de grupos de tóxicos por ingestão

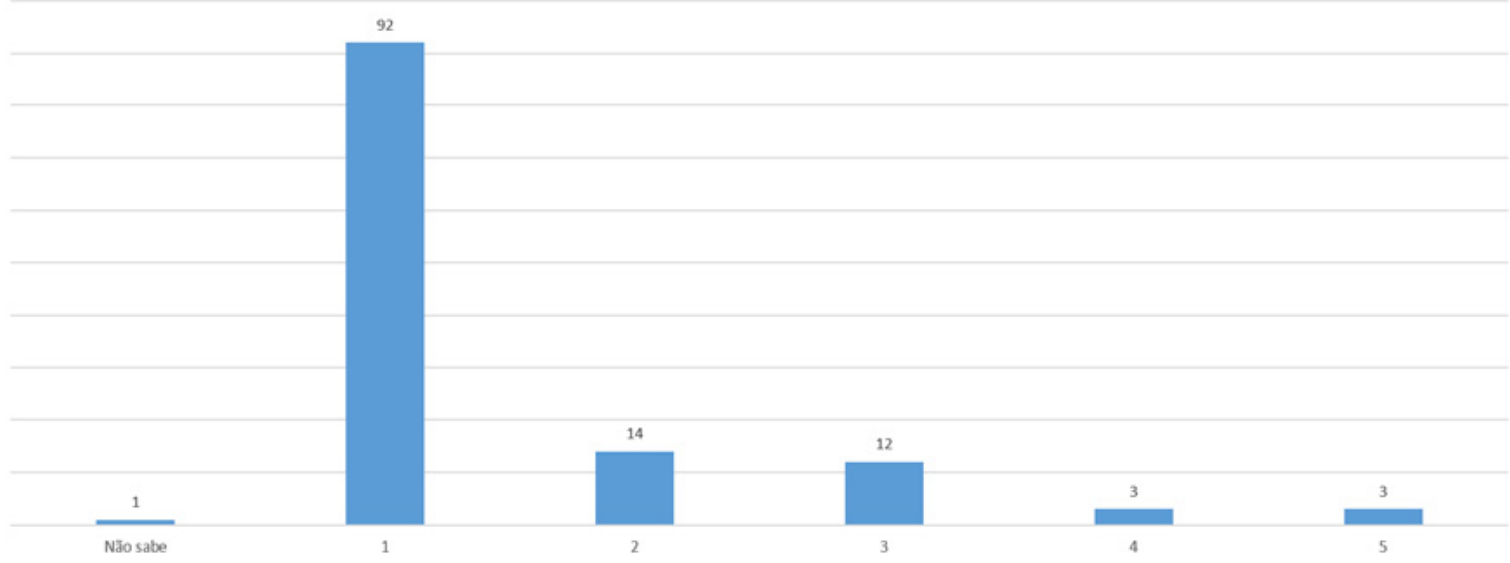



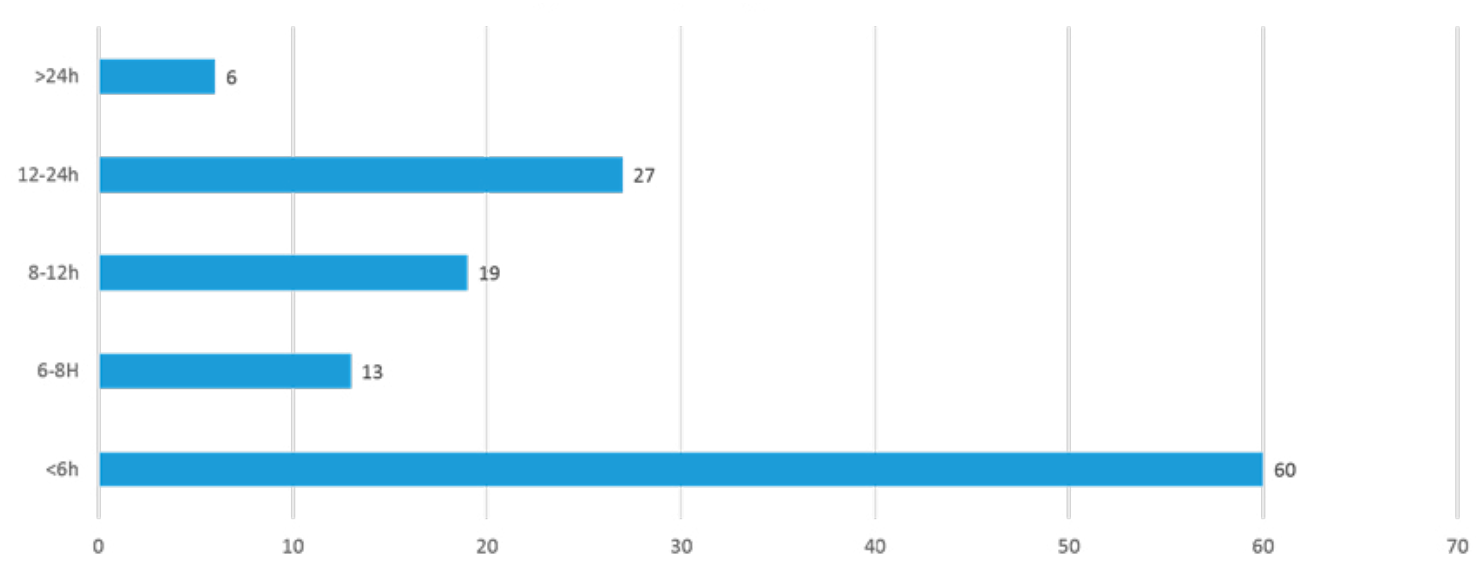

É de salientar as diferenças de padrão de tóxicos entre géneros, destacando-se o etanol como principal tóxico no género masculino e as benzodiazepinas no género feminino. Estes resultados são consonantes com o relatório de 2014 da Organizaçao Mundial de Saúde, que descreve diferenças de género no consumo de álcool. ${ }^{9}$ De acordo com este relatório, 0 consumo abusivo de álcool é o principal fator de risco de morte em homens entro os 15 e os 89 anos (com 7,6\% de todas as mortes de homens no anos 2012 a serem atribuídas ao álcool, comparativamente a 4,0\% das mortes de mulheres). ${ }^{9}$ Estas diferenças poderão ser justificadas pelo facto de, comparando os géneros quanto aos padrões de consumo de álcool, os homens tendem a ser menos abstémicos, beberem mais frequentemente e em maiores quantidades. ${ }^{9}$

A associação de vários tipos de tóxicos foi, também, concordante com outros estudos publicados, como o de Vallersnes $\mathrm{OM}$ et al referente realizado numa clínica de ambulatório em Oslo. ${ }^{2}$

Na sua maioria, os doentes foram triados como "muito urgente" ou "urgente" embora o número de complicações ou necessidade de orientação para cuidados intensivos fosse reduzido. Esta aparente discrepância poderá ser justificada pelo resultados da estratégias terapêuticas instituídas. Poderá também refletir a relevância que a triagem de Manchester atribui aos casos de intoxicação, por serem situações potencialmente fatais.

Em mais de metade dos casos não havia antídoto para 0 tóxico em questão, tendo sido instituídas apenas medidas sintomáticas. Destaca-se a percentagem de doentes em que, havendo antídoto, este não foi utilizado (n 36; 28.8\%). Estes resultados podem ser interpretados tendo conta que os tóxicos com antítodos mais frequentes foram as benzodiazepinas. 0 flumanezil não foi utilizado em 28 dos 43 casos de intoxicações por benzodiapinas (correspondente a $65,1 \%$ dos casos). A não utilização de flumazenil em quase dois terços das intoxicações por benzodiazepinas pode estar relacionada com a baixa gravidade da maioria dos quadros observados. Por outro lado, são cada vez mais conhecidos os potenciais efeitos adversos deste antídoto, que incluem convulsões e arritmias cardíacas, e fazem do flumazenil uma arma terapêutica a não usar por rotina, devendo ser ponderados os seus benefícios e riscos para cada doente..$^{10}$

Tanto 0 carvão ativado como a lavagem gástrica foram realizados na maioria dos doentes (respetivamente em 76,8\% e $72,8 \%$ da amostra). Ambas as medidas visam diminuir a absorção dos tóxicos ingeridos por via digestiva, mas a sua indicação não é universal, devendo ser ponderada caso a caso. ${ }^{5}$ Assim, o carvão ativado está contra-indicado após ingestão de agentes corrosivos ou se a via aérea não estiver protegida, enquanto a lavagem gástrica deverá estar reservada para as situações ameaçadoras de vida nos primeiros 60 minutos após a ingestão dos tóxicos, sendo também de evitar na presença de substâncias corrosivas. ${ }^{5}$

0 tempo médio de permanência no SU foi de mais de 8h, 0 que vai de encontro aos intervalos postulados para vigilância das principais complicações da maioria das intoxicações. ${ }^{1}$ Ainda assim, 60 doentes (48\%) tiveram alta até às 6 h (Figura 3), o que poderá estar relacionado com o facto da intoxicação mais frequente ser por etanol e também pelo reduzido número de complicações observadas. Por estas mesmas razões, se entende que 0 destino de alta mais frequente seja o médico de família (52,8\% dos casos). Ainda em relação aos destinos de alta (Figura 3) destaca-se a orientação para psiquiatria em $28,8 \%$ de casos, associada a ideação suicida, e a saída contra parecer médico em 9 casos.

Conclui-se que as intoxicações correspondem a uma pequena percentagem dos episódios de urgência neste SU. Atingem em média doentes jovens, na quarta ou quinta décadas de vida, mas com notória amplitude etária, destacando-se também os idosos para os quais a intoxicação acidental ganha maior peso. As benzodiazepinas são os tóxicos mais implicados e o tratamento realizado não é universal e varia caso a caso.

A assistência pré-hospitalar por VMER ou SIV ocorreu numa minoria dos casos, o que vinca a importância de conhecer as características das intoxicações mais frequentes e saber como abordá-las. 0 facto deste estudo ter sido realizado numa 
unidade hospitalar sem unidade de cuidados intensivos poderá ter influenciado a tipologia de doentes vítimas de intoxicação avaliados, uma vez que aqueles doente assistidos por equipas pré-hospitalares e com critérios de gravidade terão sido transferidos para outros hospitais.

Com este estudo foi possível conhecer a realidade do nosso SU o que facilitará a prática futura da abordagem das intoxicações.

Apesar da informação obtida, este estudo não é isento de limitações. Além de ser retrospetivo e analisar registos médicos, visa apenas 6 meses de um único serviço de urgência de um hospital pertencente ao grupo I. ${ }^{11}$

No futuro, poder-se-á obter maior informação acerca das intoxicações em Portugal através da realização de estudos prospetivos multicêntricos, eventualmente em associação com o Centro de Orientação de Doentes Urgentes do Instituto Nacional de Emergência médica. Os resultados desses estudos poderão permitir melhorar os protocolos de atuação e eventualmente criar planos de prevenção de intoxicações a aplicar na comunidade.

\section{Bibliografia}

1. Vários autores. Manual de Intoxicações. $1^{\mathrm{a}}$ edição. Edições Reanima; 2015.

2. Vallersnes $\mathrm{OM}$, Jacobsen $\mathrm{D}$, Ekeberg $\emptyset$, Brekke M. Patients presenting with acute poisoning to an outpatient emergency clinic: a one-year observational study in Oslo, Norway. BMC Emergency Medicine 2015; 15-18.

3. Vários autores. Management of poisoning. A handbook for health care workers Geneva, World Health Organization (in preparation). [Consultado 2016 Mar 22]. Disponivel em: http://www.who.int/ipcs/publications/training_poisons/guidelines_poison_control/en/index3.html

4. Kaya $E$ et al. Acute intoxication cases admitted to the emergency department of a university hospital. World J Emerg Med 2015; 6 (1): 54-59.

5. Müller D, Desel H. Common causes of poisoning: etiology, diagnosis and treatment. Dtch Arztebl Int 2013; 110 (41): 690-700.

6. Salvado ASS. Caracterização de Intoxicações Medicamentosas no Serviço de Urgência Geral do Centro Hospitalar da Cova da Beira, E.P.E. Experiência Profissionalizante na Vertente de Farmácia Comunitária e Hospitalar. Universidade da Beira Interior 2013. [Consultado 2016 Maio 2]. Disponivel em: https://ubibliorum.ubi.pt/ bitstream/10400.6/1350/1/Tese\%20M\%20Final\%20Andreia\%20Salvado.pdf

7. Mowry JM, Spyker DA, Brooks DE, McMillan N, Schauben JL. 2014 Annual Report of the American Association of Poison Control Centers' National Poison Data System (NPDS): 32nd Annual Report, Clinical Toxicology, 53:10, 962-1147.

8. Inem.pt [homepade na Internet]. Instituto Nacional de Emergência Médica. Estatísticas; 2016. [Consultado 2016 Maio 2]. Disponível em:http://www.inem.pt/PageGen. aspx?WMCM_Paginald=42030

9. Global Status Report on Alcohol and Health 2014. World Health Organization. [Consulado 2016 Maio 18]. Disponível em: http://apps.who.int/iris/bitstre am/10665/112736/1/9789240692763_eng.pdf?ua=1

10. Penninga El, Graudal N, Ladekarl MB, Jürgens G. Adverse Events Associated With Flumazenil Treatment For The Management Of Suspected Benzodiazepine Intoxication - A Systematic Review With Meta-Analyses Of Randomised Trials. Basic \& Clinical Pharmacology \& Toxicology 2016; 118: 37-44.

11. Portaria n. ${ }^{\circ} 82 / 2014$ de 10 de abril. Diário da República, 1 a $^{\text {a }}$ série — N. ${ }^{\circ} 71$ - 10 de abril de 2014: 2365. [Consultado a 2016 Maio 2]. Disponível em http:// http:// www2. portaldasaude.pt/NR/rdonlyres/05304332-B086-488E-A873-3666C5FOD6AE/0/Portaria_FAQ.pdf. 